\title{
"Mit Theater kann man etwas bewegen.“
}

Arnulf Rating im Gespräch mit Andreas Häcker über Die 3 Tornados und die Berliner Gegenkultur.

Berlin, 17. Juli 2012

\section{Andreas Häcker et Arnulf Rating}

\section{QpenEdition}

Journals

Édition électronique

URL : https://journals.openedition.org/ceg/9015

DOI : $10.4000 /$ ceg. 9015

ISSN : 2605-8359

\section{Éditeur}

Presses Universitaires de Provence

\section{Édition imprimée}

Date de publication : 1 mai 2013

Pagination : 308-321

ISBN : 0751-4239

ISSN : 0751-4239

Référence électronique

Andreas Häcker und Arnulf Rating, ",IMit Theater kann man etwas bewegen."', Cahiers d'Études

Germaniques [Online], 64 | 2013, Online erschienen am: 02 Januar 2020, abgerufen am 02 Juni 2021.

URL: http://journals.openedition.org/ceg/9015 ; DOI: https://doi.org/10.4000/ceg.9015 


\title{
„Mit Theater kann man etwas bewegen.“
}

\author{
Arnulf Rating im Gespräch mit Andreas Häcker \\ über Die 3 Tornados und die Berliner Gegenkultur \\ Berlin, 17. Juli 2012
}

\begin{abstract}
Andreas Häcker: Wie entsteht Kultur, wie entsteht Gegenkultur? Wofür oder wogegen standen Die 3 Tornados?

Arnulf Rating: Ein Lied von uns sagt das deutlich. Der Anfang war letztlich der 2. Juni 1967, das heißt die Erschießung von Benno Ohnesorg durch Karl-Heinz Kurras, der dafür keinen Tag im Knast gesessen hat - bis heute nicht. Das war gewissermaßen ein Schock, nach den ganzen Auseinandersetzungen um den Vietnam-Krieg, die deutsche Unterstützung, die ja erfolgte in Form von Geld. Unsere Generation ist noch in Trümmern des Zweiten Weltkrieges aufgewachsen, unsere Eltern waren aktiv als Soldaten daran beteiligt. Wie es der Kollege Wolfgang Neuss selig gesagt hat: „Wir sind die Kinder und Enkel von Mördern“". Dass so etwas noch einmal passieren konnte, so etwas wie die Prügelperser vor der Berliner Oper damals, die Hetze durch die Springer-Presse und der Tod von Benno
\end{abstract}

1 W. Neuss (1923-1989) war eine Kultfigur des Berliner Kabaretts der 1960er und der frühen 1970 er Jahre. Er war u. a. der Conférencier des an der Berliner Schaubühne im Januar 1969 aufgeführten Vietnam-Diskurs von Peter Weiss, wobei er die Zuschauer zu Geldspenden für den Vietcong aufrief. Als zeitweises APO-Mitglied und Gegner der Springer-Presse nahm er an Demonstrationen und sit-ins teil. 1967 veranstalte er mit den bekanntesten Liedermachern einer politischen Gegenkultur (F.J. Degenhardt, D. Süverkrüp, H. D. Hüsch) ein gemeinsames Programm. W. Biermann, der sich zu ihm auch künstlerisch bekannte, vermittelte Neuss seine erste West-Tournee.

Der Riss zwischen Kultur und Gegenkultur ging immer mehr durch Neuss hindurch, den 1943 nach einer Verwundung mit dem EK 1 ausgezeichneten Weltkriegssoldaten. Wie ein Kommentator mit Recht befand, lebte er den Protest aus, den er so lange hinausgeschrieen hatte und das bis zu seinem Tod kurz vor dem Mauerfall als drogensüchtiger, «zahnloser Späthippie » und Stadtindianer, der dennoch 1983 bei einer legendären Talk-Show zusammen mit Bundespräsident von Weizsäcker im Fernsehen auftreten konnte. « Auf deutschem Boden darf nie wieder ein Joint ausgehen ", meinte der leidenschaftliche Cannabis-Raucher zum lächelnden Hauptvertreter einer politischen Hochkultur.

Letzter Hinweis zu diesem heute Vergessenen: Neuss' Nachlass befindet sich in der Berliner Akademie der Künste. 
Ohnesorg, war ein erschütterndes Ereignis, was Handeln erforderlich machte. Das war es, was der Bewegung, der sogenannten 68er Bewegung, den Schub noch einmal gegeben hat. Ich war seinerzeit als junger Mensch, als Schüler, in Wuppertal. Wir haben eine sozialistische Schülergemeinschaft gegründet. Wir haben gegen Fahrpreiserhöhungen demonstriert. Damals war Protest eine ganz tolle Sache. Man brauchte sich nur auf die Straßenbahnschienen zu setzen und schon war das am nächsten Tag in der Zeitung. Da habe ich gelernt, wie effektiv das sein kann. Mit wenigen Mitteln konnte man viel erreichen. Mich hat allerdings immer eines gestört: die schlechte Theaterperformance im Rahmen dieser Bewegung. Wo es Aktionen waren, lief es gut. Man kann aber auch Aktionen in theatralische Formen packen. Theater eignet sich sehr gut, um Menschen in Bewegung zu bringen².

AH: Welche Theatererlebnisse waren wichtig für dich?

AR: Mich hatte Theater immer sehr fasziniert. Damals in Wuppertal, wo ich aufgewachsen bin, gab es eine der ersten Zadek-Inszenierungen: „Der Pott“. In diesem Stück geht es um eine Fußballmannschaft, es gibt einen Stürmer, er ist der Held. Er muss in den Ersten Weltkrieg, bekommt die Beine weggeschossen, wird zum Krüppel und ist nichts mehr wert. In dieser Fußballmannschaft, die da auf der Bühne auftaucht, war ein Schwarzer. Das hat Zadek so re-inszeniert. Und dieser schwarze Schauspieler nahm in einer Szene den Kopf dieses Kriegsversehrten, dieses ehemaligen Fußballstars in den Schoß und sang „Oh Haupt voll Blut und Wunden“ [Kirchenlied von Paul Gerhardt, das Bach in der Matthäuspassion wiederaufnimmt]. Das war damals wie eine Explosion, in diesem auch pietistischen Wuppertal, wo der spätere SPD-Ministerpräsident Johannes Rau herkommt. Da sind die Leute türenschlagend aus dem Theater gegangen. Und ich habe erlebt: mit Theater kann man etwas bewegen. Diese Kraft der Theaterwirkung habe ich später versucht, in die Bewegung hineinzutragen. In Berlin habe ich in den 1970er Jahren Theaterwissenschaft studiert. Wir haben uns mit unserem Professor Arno Paul im Ausland umgeschaut, wir haben mit ihm auf der ganzen Welt sozialkritisches Theater aufgespürt. Also jenseits dieser ,Hochkultur', also der bürgerlichen Kultur, gab es damals Dario Fo, den in Deutschland noch kaum einer kannte, den wir entdecken durften. Wir haben gesehen, es gibt eine Kraft des Erzählenden Theaters, die auch politisch einsetzbar ist. Das motivierte, auch so etwas zu machen. In England haben wir Kneipentheater kennengelernt, es war die Zeit von The Ken Campbell Road Show oder John Cleese, der mit den Monty Pythons im Fernsehen gearbeitet hatte. Wir haben uns gesagt, wir müssen diese Form des Humors mit politischen Inhalten verbinden und nach Deutschland tragen. Später haben wir Theater mit Lehrlingen gemacht, die uns eines Tages gesagt haben: wenn sie mit uns erfolgreich gearbeitet haben und tun, was wir anregen, fliegen sie morgen aus

2 S. den Beitrag von Charlotte Bomy in diesem Band. 
der Lehrwerkstatt raus, aber wir bekommen an der Uni einen Schein. Einen besseren Lerneffekt kann es gar nicht geben.

\section{AH: Welche Formen des Protests habt ihr begleitet?}

AR: In den späten 1970er Jahren, nachdem alle Modelle gescheitert waren, wieder eine kommunistische linke Partei, eine linke Gegenöffentlichkeit gegen Springer aufzubauen, der Wunsch eine politische Klasse für sich zu funktionalisieren oder wieder in Bewegung zu bringen, damit sich gesellschaftlich etwas ändert, kam der Sponti-Ansatz ins Spiel. Man ging nicht mehr aus abstrakten Weltrevolutionsgründen auf die Straße und organisierte sich, sondern aus der eigenen Betroffenheit heraus. Man hat gesagt: dies und dies finde ich nicht gut in diesem System - und das will ich ändern. So sind die ganzen Single-Point-Bewegungen entstanden. Man hat gesagt: hier soll jetzt kein Atomkraftwerk hin, hier wird der Stadtteil nicht so saniert, wie ihr euch das vorstellt, hier kommt jetzt keine Autobahn hin, kein atomares Zwischenlager. Es haben sich Hüttendörfer entwickelt, die Protestformen sind aus der eigenen Betroffenheit entstanden. Der Bedarf zu mobilisieren war sehr groß. Wir sind dann zu Initiativgruppen gefahren, zum Beispiel zu einer Kraftwerksbauplatzbesetzung in Grohnde. Angefangen hatte es in Brokdorf, wo ebenfalls ein Kraftwerk stand. Wir sind hingefahren und haben den Leuten, die schon in Bewegung waren auf der Straße, Geschichten erzählt, um ihnen Spaß zu machen an ihrem eigenen Einsatz. Das hing dann auch mit Selbstkritik zusammen, um mit den eigenen Widerständen in die Pötte zu kommen, das Richtige zu tun oder um Emotionalität reinzubringen. Gegen die bloße Theorie. Denn Theorie ist oft Rechtfertigung dafür, dass man nichts tut, weil man das Falsche nicht machen will. Das war der Anlass für unser Theater und wir sind dann von Initiative zu Initiative gefahren. In Grohnde sind wir aufgetreten, obwohl die uns gar nicht kannten. Wir haben als Gage ein halbes Blech Apfelkuchen bekommen. Wir wollten den Leuten Mut machen, dass es weitergeht. Es gab ja das alte Diktum von Adorno, wonach es kein richtiges Leben im falschen gebe. Aber trotzdem zu versuchen, es anders zu machen, das war der Ansatz für die alternative Kultur. Polemisch hat der Theatermann Michael Naether einmal gesagt: „Ich bin 68 aus Berlin gegangen, da waren die Studenten auf den Barrikaden, zehn Jahre später bin ich wiedergekommen, und da standen die gleichen Leute gebückt mit der Hacke und haben den Hinterhof begrünt.“ Vom „Aufrechten Gang" war also diese gebückte Haltung zurückgeblieben. Man kann sagen, dass die Bewegung auf diese Weise verkleinbürgerlicht, verkleingärtnert ist. Diese Bewegung hat aber auch schöne Sumpfblïten hervorgebracht. Leute, die die Zeit vor 1968 kennen und sie mit heute vergleichen, wissen, dass sich die Machtverhältnisse vielleicht nicht geändert haben, dass sich aber die Emanzipation des Individuums, die Lebensformen, geändert haben. Beispielsweise beim Fußball. Was sich da geändert hat, vor allem seit der Weltmeisterschaft in Frankreich. Leute wie Miroslav Klose oder Mesut Özil 
werden heute als normale deutsche Spieler angesehen. Man sagt gern, Multikulti sei gescheitert, es ist aber auf keinen Fall so. Lebenskulturell hat sich ungeheuer viel verändert. Nun ist allerdings wieder eine BiedermeierZeit eingetreten und es gibt wieder so etwas wie „Hochkultur“.

AH: 1977 gründet ihr die 3 Tornados in Berlin. Die 68er Bewegung bleibt ein wichtiger Bezugspunkt. Was ist in den 70er und 80er Jahren passiert?

AR: Berlin war für Protest eine führende Metropole. Ein äußerer Höhepunkt der Alternativbewegung war sicherlich die Hausbesetzerbewegung. Man sollte dabei aber auch die Relationen sehen: das waren knapp 300 besetzte Häuser. Bei den vielen Tausenden Häusern in Berlin war das wirklich marginal. Aber im Bewusstsein war es schon ein Höhepunkt. Denn es durfte natürlich keine „rechtsfreien Räume" geben. Und auf einmal gab es sie. Aus der Hausbesetzerbewegung ist unter anderem die Ufa-Fabrik entstanden. Sie ist ein Teil der alternativen Kultur geworden. Heute gibt es in Berlin den Karneval der Kulturen als tourismusträchtige Massenveranstaltung, das wäre damals hier gar nicht möglich gewesen. In diesem Land mit seiner ganzen Rassismus-Geschichte ist das ein schöner Erfolg. Doch die politische Kraft dieser Gegenkultur ist über Nacht mit der Maueröffnung weggespült worden. Man hat es richtig gemerkt. Wir haben kurz zuvor ein Haus besetzt, wir wollten ein Hospiz für AIDS-Kranke einrichten und wollten dafür eine Öffentlichkeit herstellen. Ende der 80er Jahre war AIDS ein wichtiges Thema. Die Besetzung lief parallel zur Maueröffnung und da hat man überhaupt keine Zeile in der Presse bekommen. Es ist eine Renaissance des Kapitalismus gekommen, der nun wieder vor einer Krise steht. Wolf Biermann sagte damals so schön, und er sagt ja nicht immer schöne Sätze: der Kapitalismus stehe wieder gut da, wie ein „scharf geschminkter Aids-Kranker“. Die Maueröffnung markierte das Ende der politischen Wirkkraft dieser Gegenkultur, kulturell wirkte sie ja weiterhin. Dieses Ende hängt auch mit der Politikwerdung dieser Alternativkultur durch die Grünen zusammen. Bei den Tornados haben wir immer gesagt: „Wer seine Rechte delegiert, hat seine Linke liquidiert“. Die Grünen haben die Alternativbewegung, die Protestbewegung in dem Parlamentarismus beerdigt.

AH: Zu den Grünen kommen wir später noch einmal zurück. Wer sind die Mitglieder der 3 Tornados?

AR: Günter Thews und ich haben Theaterwissenschaften studiert und wollten unseren Erfahrungshorizont mit Dario Fo oder mit dem London Fringe Theater ausbauen. Wir nannten uns schon „Die 3 Tornados“ als wir noch zu zweit waren. Wir fühlten uns als Teil der Berliner "Spaßguerilla“. Das war Anfang 1977, da haben wir in Kneipen gespielt und haben gesehen, dass wir etwas brauchen, um die Aufmerksamkeit der Leute zu gewinnen. 
Wir hatten einen Bekannten, ein Philosophiestudent, der bei Feten seine Quetsche auspackte. Der spielte dann zum Beispiel die Erkennungsmelodie der Fernsehserie „Flipper“. Hans-Jochen Krank war Hesse, den haben wir gefragt und er hat mitgemacht. So kam es zur Urformation: Hans-Jochen Krank an der Quetsche, Günter Thews und ich. Nach dreieinhalb Jahren ging dann Hans-Jochen Krank und es kam Holger Klotzbach.

AH: Das umgedichtete Lied von Flipper ist ein gutes Beispiel für eure Arbeit. Die 3 Tornados nahmen gerne Elemente der Mainstream-Kultur ins Programm auf und luden sie subversiv auf. Kann man das so sagen?

AR: Ja, es gab ja damals so eine Sehnsucht nach Volkstheater. Wir aber wollten nie so richtig volkstümelndes Theater machen. Natürlich war für uns von Interesse, wie Theater im Volk, in der breiten Öffentlichkeit wirken kann. Wir haben starke Erfahrungen gemacht. Für unser Lehrlingstheater hatten wir seinerzeit in der Disko Werbung gemacht. Um 22 Uhr haben wir die Musik ausgestellt und haben Theater gemacht. Da wussten wir, dass wir sehr gute Pointen im Köcher haben mussten, sonst wird man nach 30 Sekunden ausgebuht. Oder auch später, als wir an der Uni gespielt haben bei den Streiks und Protesten, wo es vorne immer eine festgelegte Rednerliste gab mit den verschiedenen Kampfgruppen, K-Gruppen, unabhängigen Gruppen, von Undogmatischen, von Frauen. Die Reihenfolge war fest und kanonisiert. Wenn man sich einfach so auf die Rednerliste setzte oder das Podium besetzte, dann musste man sofort alle Gesinnungspolizisten der fünf Parteien da vorne zum Schweigen oder - besser - zum Lachen bringen. Es war klar, wir mussten an etwas anknüpfen, was alle kennen. Das Flipper-Lied entstand zu der Zeit, als in Berlin der Delphinzirkus Conny's Flippershow gastierte. Da fiel uns Hans-Jochen Krank ein; er kam bei uns mit der Taxe vorbei, mit der er sein Studium finanzierte, packte seine Quetsche aus und die Noten, da haben wir dann das "Lied zum Kontaktbereichsbullen" umgetextet und mit der Taxe ging es dann sofort in die nächste Kneipe. Das war ein paar Tage vor dem ersten Mai. Und am 1. Mai wurde das Lied auf der Demo gesungen. Da waren wir glücklich, wir haben gesehen, so geht's, das ist der Weg (lacht).

AH: Im Jahr 1977 braucht der Protest in Berlin anscheinend viel Ordnung. Manche Fraktionen fordern Tagungsordnungspunkte und Geschäftsordnungen. Wo kann man da Die 3 Tornados situieren?

AR: Wir haben genau dies durchbrechen wollen. Wir haben uns immer sehr daran gestört, wenn praktisch im Streik an der Uni gegen neue Gesetze oder Studienordnungen solange debattiert wurde, bis der Plan, etwas zu tun, zerredet war. Und genau in diesem Moment dachten wir: Theorie gibt es genug, die müssen wir nicht liefern. Wir müssen die Stimmung zusammenfassen und auf den Punkt bringen, die Rednerliste abkürzen und sagen: „So - jetzt Aktion! Wir wissen jetzt genug. Unser Defizit ist das 
Handeln." Da muss jemand kommen, der das so sagt, wie es alle verstehen können und nicht noch einmal eine Theoriedebatte entsteht. Wir mussten von unten kommen, und manchmal das Intellektuelle weglassen. Wir sagten bei den Tornados immer, wir wollen Theater machen, aus dem der Zuschauer oben mit erweitertem Bewusstsein und unten mit nasser Hose rauskommt. Das haben wir versucht, das war die Strategie und das hat auch funktioniert auf dem Marktplatz, bei besetzten Häusern, bei der Autobahn, auch im Konzertsaal und später auch im Kabarett.

AH: Ihr spieltet in Unihörsälen, in Kneipen, auf Straßendemos. Wer kam zu den 3 Tornados?

AR: In Berlin haben wir das ernst genommen. Wir haben uns geschworen, in Berlin nur auf politischen Veranstaltungen zu spielen. Natürlich wurden wir in Westdeutschland überall eingeladen und sind dort auf allen möglichen Veranstaltungen, auch auf ganz normalen Konzerten aufgetreten. Wir wollten ja kein Kabarett machen, Kabarett war für uns ein Schimpfwort. Wir gingen dahin, wo die Leute etwas bewegen wollten, auf politische Veranstaltungen. Zum Beispiel auf den TUNIX-Kongress ${ }^{3}$. Da war selbstverständlich, dass wir dabei waren. Der TUNIX-Kongress, auf dem sich auch eine Arbeitsgruppe bildete für eine linke alternative Tageszeitung, aus der dann die taz entstanden ist, war ein Reflex auf den Herbst 1977, auf die Lähmung der Öffentlichkeit durch die RAF, durch den Umgang mit der RAF. Die Wirkung der RAF passte, meiner Ansicht nach, politisch den Regierenden gut in den Kram, um die Linke zu spalten, um sich in die Debatte hineinzufressen. Wir haben gegen diese Spaltung, gegen diese Falle debattiert, wir suchten wirklich nach Alternativen. Hier taucht auch wieder der Begriff auf: alternativ. Der TUNIX-Kongress und die Entstehung der taz zeigten, dass wir den Wunsch hatten, die Theoriedebatte zu beenden und der Debatte um die Rolle, in die uns die Mainstream-Gesellschaft drängte, zu entrinnen. Und Alternativen zu entwickeln. Viele haben damals gesagt, dass das so nicht gehe. Mit ,alternativ' verband man gern Leute, die das taten, was sie nicht gelernt hatten, die nicht wissen, was sie tun, die unprofessionell waren. Man kam zu spät, ein gewisses Image wurde ja gepflegt. Aber aus der Alternativbewegung ist, im Nachhinein gesehen, einiges Vernünftiges entstanden.

AH: Der TUNIX-Kongress fand 1978 statt. Dort hat auch Peter Glotz, damaliger Wissenschaftssenator der SPD in West-Berlin, seine Theorie der zwei Kulturen dargestellt. Hast du seinen Vortrag im Hörsaal mit verfolgt?

3 Der Tunix-Kongress, der als Antwort auf den „Deutschen Herbst“ 1977 viele Gruppen der « Sponti-Bewegung » in der Berliner Technischen Universität vom 27. bis zum 29. Februar 1978 zusammenführte, markierte den Anfang einer Neuorientierung des 68er Protests und der Autonomen- und Alternativbewegungen. 
AR: Nein. Wir haben anderswo auf dem Kongress gespielt und andere Seminare besucht. Aber ich kann mir gut denken, was er da gesagt hat. Er war der Theoriepapst der SPD. Papst hin oder her: man kann ja immer froh sein, wenn einer bei der SPD noch Theorie kann statt nur wursteliger Praxis. Die Theorie der zwei Kulturen war wohl eher ein Holzwegargument ${ }^{4}$.

AH: Kommen wir zur Dramaturgie der 3 Tornados. Wenn man in der Disko oder auf Demos gegen Atomkraftwerke Theater macht, braucht man wohl besondere Darstellungsformen. Wie wichtig ist Musik, Lachen und Humor für euch gewesen?

AR: Lachen war sehr wichtig. Für uns war Deutschland ein Humorentwicklungsland. Was nicht zuletzt daran lag, dass während des Faschismus die Juden ermordet wurden und so die jüdische Intelligenz verlorenging, die ja immer eine kritische und humoristische Intelligenz war. An diesem Defizit mussten wir arbeiten. Dramaturgisch ging es darum zu überzeugen, zur Handlung zu überzeugen. Das ging am besten mit Humor. Humor ist entwaffnend. Es ist schön, seine Gegner, auch seine Gegner auf theoretischem Feld, zum Lachen bringen. In der Politik, in der Parteienlandschaft gibt es ja die Steigerung: Freund, Feind, Parteifreund. Es war immer so, dass die, vor allem im linken Spektrum, die sich politisch besonders nahe standen, sich auch immer selbst die größten Gegner waren. Und in diesem Kampf wollten wir dazwischen gehen. Da ist es von Vorteil, wenn man alle erst einmal zum Lachen bringt. Da kann man auch gelegentlich in eine triviale Kiste greifen, auch auf die Gefahr hin, dass die Leute "unter ihrem Niveau" lachen. Wir wollten wirklich aktuelle Themen ansprechen, die nicht über die Köpfe der Leute hinweggingen. Wir gingen von unserem eigenen Standpunkt, von unserer eigenen Betroffenheit aus. Das bedeutete auch, sich selbst zum Gegenstand des Humors machen. So kann man Autorität gewinnen, auch etwas anders zu sagen. Und zusätzlich hilft die Musik. Wir hatten eine fast mittelalterliche Theaterform und insofern eine Volkstheaterform gewählt. Wir mussten uns den Bühnenraum immer selbst suchen und schaffen, wir wollten beweglich sein. Damals gab es ja auch die Idee, große Theaterkollektive zu schaffen. In den USA gab es das Living Theater oder das Bread \& Puppet. Wenn man mit zwölf Leuten Theater macht, dann kommt man intern wieder zur Debattenkultur zurück. Wir wollten aber auch kein großes Kollektiv mit autoritären Strukturen. Als kleine Truppe waren wir doch sehr agil und mobil. Das entsprach auch unserer materiellen Organisation. Hans-Jochen hat uns mit der Taxe herumgefahren, jeder hatte einen Koffer mit Klamotten, Hüten und Brillen. Mit den angedeuteten Verkleidungen sind wir dann in die Figuren geschlüpft. Die

4 P. Glotz verfocht innerhalb der SPD einen « erweiterten Kulturbegriff », der nicht nur die Kluft zwischen Elite- und Populärkultur möglichst verringern sollte, nicht aufs „MusischKünstlerische" begrenzt war und die verschiedenen Formen einer wissenschaftlichen Kultur mit einbezog. Darin war aber von „Gegenkulturen“ keine Rede. 
Quetsche diente dazu, nach einer Szene mal wieder zu entspannen. Und so entstand eine eigentlich ganz simple Szenenfolge: Lied, Szene, Lied, Szene... Am Anfang sind die Szenen daraus entstanden, dass wir am Freitag bei einer Bürgerinitiative für die Stadtteilsanierung am Chamisso-Platz gespielt haben, am Samstag waren wir in Brokdorf auf einer Demo gegen Atomanlagen, und am Sonntag waren wir bei der Bürgerinitiative Westtangente, die den Stadtautobahnausbau verhindern wollte. Die Bürgerinitiative Westtangente hatte den damals belachten, aber heute anerkannten Satz geprägt: jede Straße erzeugt Verkehr, wir müssen das Primat des Autos in der Stadt umdefinieren. Das fanden wir genial und so haben wir diese Initiativen unterstützt und von deren Problemen erfahren, wir haben diese Probleme in Szenen verwandelt. Und natürlich haben wir gespielt, wenn sie im Kiez ein Fest gaben, damit sie auch Zulauf hatten. Daraus war unser Bühnenprogramm entstanden. Wir waren so etwas wie eine lebende Stadtzeitung. Weil wir den Leuten in Schwäbisch Gmünd erzählten, was in Gorleben los war, und in Gorleben, dass die Leute im Schwäbischen die Remstalautobahn verhindert haben. Wir waren die Alternative zum später entstehenden Kabelfernsehen.

AH: Die 3 Tornados haben auch Linke, Leute aus dem eigenen Lager, gerne kritisiert. Es gibt da zum Beispiel den Sketch, in dem ein grüner Bundestagsabgeordneter einen Mann von der Basis ins Restaurant einlädt. Worum geht es in dem Sketch?

AR: Die Grünen haben ja angefangen mit Basisdemokratie. Jeder durfte mitreden und mitentscheiden, unabhängig davon, ob jeder noch nach dem fünften Bier zurechnungsfähig war. Sicher hatten die Grünen einige Vorzüge. Es war es von Vorteil, wenn man jemanden hatte, der sich mit Verwaltung auskannte, der Rechtsanwalt war, der eine Demo anmelden konnte oder einen aus dem Knast holen konnte, es war natürlich auch vernünftig. Das zeigt ja auch die Geschichte der Hausbesetzer, wenn da erfolgreich verhandelt wurde. Einige besetzte Objekte wurden an die Besetzer übergeben, was übrigens komischerweise nicht zu neuen Eigentumsformen geführt hat, sondern lediglich zu neuen Eigentümern. Irgendjemand hat Reibach gemacht. Über einen Kollektivakt ist dann wieder privatisiert worden. Aber die Grünen haben eben das geleistet, dass sie die Alternative in die bestehende Demokratieform überführt haben. Und da kam der Punkt irgendwann ziemlich schnell, dass grüne Abgeordnete, die zwar brav der Partei die Hälfte ihres Abgeordnetengeldes abgegeben haben, andererseits dennoch in einer privilegierten Position waren aus der heraus sie z.B. von Waffenlobbyisten einen kleinen Kredit bekommen konnten wie Cem Özdemir. Das ist quasi logisch, wenn jemand Macht bekommt - und der Parlamentarismus ist eine

5 Die « lebenden Zeitungen », die in Sowjetrussland als theatralische kleine Form der Nachrichtenübermittlung auch die rückständigsten Volksschichten erreichen sollten, waren auch nach einer Deutschlandtournee der sowjetischen Blauen Blusen ein beliebtes Mittel der Weimarer Agitprop geworden. 
Macht. So werden die, die im Parlament sitzen und die Macht haben, gerne mal zum Essen eingeladen, werden hofiert, bekommen andere Kontakte. Dieser Konflikt zwischen einem Mann von der Basis, der damals nach Bonn kommt, und seinem linken grünen Abgeordneten, der ihn ins Restaurant einlädt, dieser Kampf der Kulturen war natürlich genüsslich darzustellen.

AH: Die 3 Tornados nehmen nicht nur die Grünen aufs Korn, auch die Institution Fernsehen wird parodiert. Trotzdem schafft ihr es 1979 sogar, mit fast einer Stunde Programm in eine Liveübertragung von der Internationalen Funkausstellung in Berlin zu kommen.

AR: Heute kann man es sich gar nicht mehr vorstellen, diese Hysterie in den Sendern, die Angst hatten. Brechts Radiotheorie war ja nicht nur bei den Linken im Kopf, sondern auch bei den Herrschenden ${ }^{6}$. Es gab hier beim SFB, beim Sender Freies Berlin, eine Zeit, wo unten beim Pförtner ein Polizeiposten war, der verhindern sollte, das irgendwelche Leute die Nachrichtenredaktion besetzten, um dann irgend etwas durchzusagen. Wir waren damals auf der Seite der Besetzer. Berliner Künstler? Das waren für den SFB damals Hildegard Knef, Harald Juhnke, vielleicht noch Günter Pfitzmann. Und das war es. Eine andere Kultur hat dieser Sender gar nicht gesehen. Wir sind da durch Zufall schräg reingekommen. Zur Internationalen Funkausstellung mussten alle ARD-Redaktionen Live-Veranstaltungen produzieren, damit letztendlich Fernsehgeräte verkauft werden konnten. Inhalt, „,ontent“ wie man heute sagt, musste da geliefert werden. Ein Redakteur hat uns dabei im Kinderprogramm untergebracht, irgendwie hatte da keiner so genau hingeguckt, bis dann das Drehbuch in der Kantine liegenblieb. Das ging sofort an die Rechtsabteilung. Darauf mussten wir der verantwortlichen Programmleiterin jede Pointe einzeln erklären. Es wurde verfügt, so etwas nicht einfach auf die Menschen loszulassen, da müsse eine Moderatorin hin. Die Moderatorin, die dann ausgewählt wurde, nette Frau, hatte aber höllische Angst. Wir haben uns hinter der Bühne geeinigt: sie moderiert am Anfang und am Schluss, wir spielen durch, und dann ruft das Publikum sowieso nach Zugabe. Die Verantwortlichen der ARD saßen in der

6 Brechts Radiotheorie, die in den Jahren 1927-1932 entstand, nahm sich vor, Rundfunkzuhörer an den Sendungen aktiv zu beteiligen, um die „eingreifende“ Funktion der Kunst zu verstärken: „Der Rundfunk ist aus einem Distributionsapparat in einen Kommunikationsapparat zu verwandeln. Der Rundfunk wäre der denkbar großartigste Kommunikationsapparat des öffentlichen Lebens, ein ungeheures Kanalsystem, das heißt, wenn er es verstünde, nicht nur auszusenden, sondern auch zu empfangen, also den Hörer nicht nur hören, sondern auch sprechen zu machen und ihn nicht zu isolieren, sondern in Beziehung zu setzen. Der Rundfunk müsste demnach aus dem Lieferantentum herausgehen und den Hörer als Lieferanten organisieren." (Bertolt BRECHT, Werke, Große kommentierte Berliner und Frankfurter Ausgabe, Bd. 21, Berlin-Weimar / Frankfurt am M., Aufbau / Suhrkamp, 1992). Brecht schrieb 1929 Der Ozeanflug, ein Hörspiel als „Rundfunkexperiment“.

1970 bezog sich H.M. Enzensberger in „Baukasten zu einer Theorie der Medien“ (Kursbuch 20) auf Brechts Radiotheorie. 
ersten Reihe. Wir hatten unter anderem in einem Lied zum Schwarzsehen aufgerufen. Nachdem wir eine Zugabe machten, die als geschmacklos bewertet wurde und in der es gegen die SPD ging, sah alles erst nach einem Skandal aus. Schließlich hat man sich aber gesagt, dass das doch Kabarett und nicht ganz unlustig war und es wurde sogar noch einmal wiederholt. So hat sich das beruhigt.

Ganz anders war es dann, als wir nicht mehr nur auf politischen Veranstaltungen in Berlin aufgetreten sind. Da haben wir uns gesagt: die müssten uns im SFB in der Abendschau auch mal ankündigen. Die Abendschau hätte zwar über alle möglichen Friseursalonmeisterschaften berichtet, aber nie über uns. Weil wir nun nicht redaktionell ins Fernsehprogramm kamen, sagten wir uns: „Wir kaufen uns das Fernsehen.“ Und da haben wir geguckt, was die kleinste Einheit Werbezeit kostet, die man kaufen kann. Wir haben einen kleinen Spot gemacht bei einer alternativen Medienkooperative, die damals Videos produzierte. Der Spot dauerte vielleicht acht Sekunden und begann so: „Hey, was ist denn da los? Das sind Die 3 Tornados!" Das war unser Eröffnungslied. Im Umschnitt wurde ein Polizist gezeigt, der mit Tomaten und Eiern beworfen wurde. Zum Schluss sagte er: „Endlich in Berlin.“ Und guckte hinter seiner Zeitung hervor. Das war Werbezeit, die wir bei der SFB Werbefernsehen \& Co. KG gekauft hatten. Der SFB, so wurde behauptet, hatte aber aus inhaltlichen Gründen abgelehnt, diesen Film zu zeigen. Gegen diese Verfügung haben wir geklagt. Wir haben argumentiert: wir machen reine Wirtschaftswerbung, es geht uns darum, Karten zu verkaufen, und da muss auch etwas vom Charakter unseres Produktes im Werbespot drin sein. In Zahncremewerbung ist auch ein Zahnarzt dabei, ebenso kommt bei uns ein Polizist vor. Das haben wir auch vor Gericht gewonnen. Der Sender wurde verpflichtet diesen Werbespot zu bringen. Für uns war dabei der größere Gag, dass die ganze Presse darüber berichtete. In der Schürze einer Berliner Boulevardzeitung stand ganz groß: „Gericht erlaubt Steinwürfe auf Polizisten...“ und klein gedruckt darunter „... im Werbefernsehen".

AH: Die Aufmerksamkeit der Massenmedien war nun da.

AR: Das war damals so. Mit solchen Mitteln konnte man etwas bewegen. Das geht heute sicherlich auch gelegentlich. Zumindest bekommt man Öffentlichkeit und Debatte.

AH: 1980 gab es ein Krippenspiel von Euch mit einem ziemlich dicken Nachspiel. Was war denn da los?

AR: Beim WDR gab es die Jugendsendung „Radiothek", die abgesetzt wurde. Das kann man sich heute gar nicht mehr vorstellen: damals war das Programm ziemlich überaltert. Beat-Musik im öffentlich-rechtlichen Sender 
galt gemeinhin noch Anfang der 1970er Jahre als aussätzig. Kam auch nicht vor. Später wurden „Jugendwellen“ gegründet, die für zwei oder drei Stunden ein Programmfenster hatten. Im SFB gab es "SFBeat" und beim WDR die „Radiothek“. Diese Sendungen griffen nicht nur die aktuelle Musik, sondern auch das gesellschaftliche Verständnis jüngerer Hörer auf. Das war ein Ärgernis im Öffentlichen Rundfunk. Die Privaten gab es damals noch gar nicht. Der WDR war sowieso als Rotfunk (guillemets?) verschrien und wurde politisch bekämpft. Irgendwann wurde nun diese Sendung, die „Radiothek“, geopfert. Die „Radiothek“-Redaktion, die sehr aufmüpfig war, hatte eine mehrstündige Live-Abschiedsveranstaltung organisiert, zu der wurden wir auch eingeladen. Der spätere Wellenchef von WDR 5 und heutige Rundfunkchef hatte uns eingeladen und gebeten, „Maria und Joseph" zu spielen. Das alternative Krippenspiel war ein Nebenprodukt einer unserer Tourneen durch Bayern, als gerade der Papst zu Besuch war. Überall im Fernsehen, in jeder Pension, in jedem Wirtshaus lief der Papst. Wir sagten uns: das kann doch nicht sein. Der darf ins Fernsehen, wir nicht, nur weil vor zweitausend Jahren mal ein uneheliches Kind geboren wurde. Da haben wir uns entschlossen, den Leuten zu erzählen, wie diese Geburtsgeschichte wirklich war. Josef, der Zimmermann, und Maria liegen abends im Bett. Josef möchte bumsen, aber Maria wehrt ihn ab: „Heute nicht, Schatz!“ Um schließlich zu gestehen, dass sie ihre Tage nicht bekommen hat. Diese Geschichte haben wir erzählt, in einer sehr flapsigen Sprache. Sicher war das Thema Sexualität vor allem in Verbindung mit Religion damals noch provokanter als heute. Wir haben das Stück im Auto geschrieben, es war thematisch ein wirkliches Nebenprodukt für uns. Vor der ersten Aufführung in Aachen haben wir den Veranstalter gebeten, ob er ein Bettlaken hätte für das gemeinsame Bett und ein blaues Tuch für die Maria. Wir hatten nie damit gerechnet, dass das Stück so bei katholischen Kreisen einschlägt. Schüler haben das Stück in Schülerzeitungen abgedruckt und sind anschließend dafür von der Schule geflogen. Bei der Radiothek-Sendung war der Beitrag ursprünglich für den Nachmittag vorgesehen, so ca. 17 Uhr. Und es wurde immer weiter nach hinten verschoben, wir wussten nicht wieso. Die Leute vom WDR hatten sich das Stück ja gewünscht, wir hatten auch noch anderes im Programm, das uns viel wesentlicher war. Wir mussten immer länger warten, vor uns spielte dann auch $\mathrm{BAP}^{7}$, einer ihrer ersten Auftritte. Nach 22 Uhr haben wir das dann gemacht, alle haben sich gefreut und so ging es live über den Sender. Erst fünf Tage danach hat sich eine Bürgerinitiative aus dem katholischen Soest formiert. Angeführt wurde die Gruppe, die sich in ihrem Glauben verletzt fühlten, von einer Frau, die wir auch später besucht haben, bei Kaffee und Kuchen. Diese Gläubigen haben sich ihre Glaubensverletzung als Tonband von der Sendung mutwillig reingezogen und Unterschriften gesammelt. Es waren also Leute, die diese Sendung gar nicht im Radio gehört

71976 gegründete Pop-Rock-Gruppe, die im Stil von Bob Dylan und den Rolling Stones des Öfteren soziale Probleme aufgreift. 
hatten. Sie wurden nach dem Gottesdienst gebeten, sich das anzuhören, um sich zu empören. Irgendwann sah sich die Staatsanwaltschaft gezwungen, einen Prozess zu eröffnen. Ein verantwortlicher Redakteur im WDR, eigentlich waren es drei, wurde ins Musikarchiv strafversetzt. Es hat niemand ausgesprochen, aber wir hatten faktisch während fünf Jahren auf allen deutschen Stationen Sendeverbot.

AH: Es kam zum Prozess vor dem Amtsgericht Köln.

AR: Wir hatten ja Theaterwissenschaft studiert und argumentierten vor Gericht, man könne den Gehalt der Szene aus dem Text allein ja nicht ablesen. Theater ist in der Umsetzung mehr als der reine Text. Wir müssten das jetzt vor Gericht vorführen. Wir das Stück vor Gericht vorgespielt. Bei Strafandrohung von drei Tagen Ordnungshaft durfte das Publikum nicht lachen... wir wurden verpflichtet, das allerdings im Angesicht der Richter zu spielen, die dann betont desinteressiert nach unten schauten. Wir hatten zuvor auch die örtliche Zuständigkeit des Gerichts gerügt. Wir hatten dazu festgestellt, dass die einzigen Zeugen, die etwas zu dem Gespräch zwischen Maria und Joseph sagen könnten, Maria und Josef selber seien, diese aber bekanntlich leider seit längerem verstorben und somit nicht als Zeugen vor diesem Gericht erscheinen könnten. Es gebe allerdings eine Instanz, die alle Verfahrensbeteiligten versammeln könne und das sei das Jüngste Gericht. Wir entzögen uns damit keiner Strafe, denn das Jüngste Gericht verfüge ja bekanntlich über zahlreiche Sanktionen. Deshalb baten wir um Abgabe des Verfahrens ans Jüngste Gericht. Der Antrag war formal richtig gestellt. Wir hatten dazu unter anderem Otto Schily und Hans-Christian Ströbele als Berater $^{8}$. Das Gericht war formal gezwungen, sich zur Beratung über diesen Antrag zurückzuziehen erklärte sich aber dann doch für zuständig. Wir plädierten auf Freiheit der Kunst, hatten auch ein Gutachten von Udo Lindenberg vorliegen, der sagte, wir drückten den biblischen Inhalt lediglich aus in der Sprache junger Menschen. Wir wurden dennoch verurteilt, weil wir nach Meinung des Gerichtes die Freiheit der Religionsausübung beeinflusst hätten. Artikel 5, Freiheit der Kunst, gestand uns das Gericht nicht zu, wir hätten vielmehr gegen Artikel 4, Freiheit der Religionsausübung verstoßen. Was ja vollständiger Quatsch war: wir hatten niemanden etwa daran gehindert, in eine Kirche zu gehen und sind auch in keine Kirche hinein, um dort den Gottesdienst zu unterbrechen. Wir haben Revision eingelegt, es kam zum nächsten Verfahren. Da gab es eine der schönsten theatralen Aufführungen, die ich je in meinem Leben gesehen habe. Und zwar wurde zur Beweiserhebung das alte Verfahren per Aktenvorlesung in das neue Verfahren eingebracht. Ein beisitzender Richter musste daher das Stück

8 Schily und Ströbele waren in den 1970er Jahren Rechtsanwälte, die der APO nahe standen und u. a. RAF-Mitglieder verteidigten. Sie gehörten später zu den Gründungsmitgliedern der Partei Die Grünen. Ströbele ist heute noch Bundestagsabgeordneter der Grünen. Schily wechselte zur SPD über und wurde Bundesinnenminister. 
Maria und Josef vorlesen, mit verteilten Rollen. Und das hat er sehr verschämt, distanziert, episch verfremdet gemacht, wurde dabei leicht rot. Ich konnte mich nicht halten vor Lachen. Das war sehr, sehr komisch. Wenn wir keine Freiheit der Kunst reklamieren könnten, sagten wir uns in diesem Verfahren: Wir sind doch selber eine Religionsgemeinschaft. Wir sind drei Menschen, wir sind eines Glaubens: wir glauben, dass diese Geschichte zwischen Maria und Joseph genau so passiert ist. Beim abendlichen Zähneputzen sei eines Tages aus meinem Wasserhahn Wein gekommen, der Erzengel Gabriel sei mir erschienen und habe gesagt: „Es war genauso, wie ihr es erzählt." Meine Kollegen, denen ich das am nächsten Morgen mitgeteilt hatte, haben mir sofort geglaubt und seitdem sind wir eine Glaubensgemeinschaft, welche diese Geschichte ihrem Publikum verkündet. Wir baten den Richter, unseren Glauben in Schutz zu nehmen vor der Verfolgung durch den Staatsanwalt. Das hat der Richter aber nicht getan, und wir wurden wieder verurteilt.

AH: Der Ordner mit den Prozessakten im Deutschen Kabarettarchiv in Mainz ist ziemlich dick. Vielleicht können wir die Geschichte zum Krippenspiel ein bisschen abkürzen? Gab es nach mehreren Instanzen am Ende Freispruch?

AR: Ja. Wir sagten in späteren Prozessen, dass es eigentlich ziemlich unwürdig wäre, gerade mit Blick auf die deutsche Geschichte, wenn eine so große Religionsgemeinschaft wie die katholische Kirche so ein starkes Schutzbedürfnis beanspruche. Am Ende gab es dann einen formalen Grund, aus dem man uns schließlich frei gesprochen hat.

AH: Martin Bayer, der euren CD-Kollektor bespricht, schreibt: „Die 3 Tornados, das war im Kabarett, was Ton Steine Scherben für die Musik war: eine Institution und trotzdem keine ehrfurchtgebietende Sache, vielmehr etwas sehr Lebendiges." Gab es auch Beziehungen zwischen den 3 Tornados und den Leuten von Ton Steine Scherben?

AR: Ja, wir haben uns sehr gemocht. Wir sind im Tempodrom - 1980 als alternative Spielstätte direkt an der Mauer gegründet - zusammen aufgetreten. Wir haben ja das Tempodrom mit aufgebaut, es sollte ein für alle zugängliches Haus sein, ein Ort der Alternativkultur. Da haben wir bei der Eröffnung und bei den ersten Revuen gemeinsam mit den Ton Steine Scherben gespielt. Wir hatten bis zuletzt mit Rio Reiser, dem Frontmann der Gruppe und teils bis heute mit den anderen Freundschaften. Wir haben auch gemeinsam etwa in Hamburg gespielt und bei Kampnagel eine Halle mit besetzt ${ }^{9}$. Die Scherben-Songs werden ja bis heute auf fast jeder Demo

9 Kampnagel ist eine ehemalige Maschinenfabrik in Hamburg, die seit 1982 als Bühne für zeitgenössische Theateraufführungen und Performances dient. 
gespielt, aber in der nicht etablierten Szene waren damals Live-Auftritte viel gefragter.

AH: Mit den 3 Tornados hast du früher linkes Straßentheater gemacht. Heute bist du Kabarettist, seit kurzem spielst du in Videoclips für das „Manager Magazin“, das ja nicht unbedingt von jedem guten Sozialisten gelesen wird.

AR: Erstmal würde ich mich auch nicht als guten Sozialisten bezeichnen, sondern eher als Anarchisten. Und zwischen Anarchie und Finanzmärkten ist eine gewisse Nähe da. Ähnlich wie wir damals ins Fernsehen reingerutscht sind, ist es heute beim „Manager Magazin“. Das hat seine Internetseiten renoviert und dort gibt es nun auch bewegte Bilder. Also sagten sie: filmen wir doch ein paar witzige Kommentare zu dem, was in der Finanzwelt los ist. Und mit dem Projekt wird ein Redakteur beauftragt, der wieder jemanden beauftragt und der hat Kontakt zu einer bestimmten Szene von Kabarettisten. Und dann wird man gefragt und sagt: „Warum nicht?" Solange das so ist, das man machen kann, was man will, ist das genauso ein Versuch wie damals, um eine breite Öffentlichkeit zu erreichen und die Leute zum Handeln zu bewegen, was in diesem Fall dringend notwendig ist.

AH: Du schaust auch nach dem Nachwuchs. Gibt es heute noch Gegenkultur? Gibt es heute noch Künstler im Kabarett, die sich trauen gegen Biederkeit Programm zu machen?

AR: Es gibt diesen Begriff der Gegenkultur ja gar nicht mehr, weil alle "dafür" sind. Es ist immer so, dass gewisse Probleme in einer Kultur oder in einer sozialen Gesellschaft gelöst werden. Ein paar andere Probleme bleiben. Das Problembewusstsein artikuliert sich immer in neuen Formen. Im Moment geht ein Trend gerade vom Kabarett weg, hin zu Lesebühnen. Der Prozess ist schon lange im Gange, schon seit den 90er Jahren. Die mediale Öffentlichkeit hat es nie kapiert und verleiht weiterhin noch Kleinkunstpreise. Zunehmend wird es entdeckt, wie zum Beispiel Marc-Uwe Kling oder Horst Evers Die beiden sind inzwischen Buchmarktgrößen. Früher haben die Verleger immer gesagt, das ist eine zu kleine Form, mit der können wir gar nichts anfangen, wir brauchen Romane. Jetzt stellen sie fest, dass das reichlich Leser findet. So wächst immer etwas nach. Gerade Berlin ist ein wahres Biotop, es geschieht so viel in der Literatur und auf den Lesebühnen. Es wird vom Feuilleton immer gern die Galerieszene gesehen. Die Lesebühnenszene ist vielleicht eine nicht direkt politisch formulierende Szene, aber doch sehr personenbezogen. Man argumentiert von der eigenen Erfahrung aus, das ist eine Erzählweise, die das Individuelle und Private als Befindlichkeit artikuliert, das ja auch politisch ist. Sie stellt damit das Bewusstsein einer neuen Generation vor. Die Kultur, die wahrgenommen wird, ist ja meist Mainstream, der Kommerz. Fast alle Fernsehproduktionsfirmen sitzen heute 
in NRW, weil es dort Förderung gab, worum sich etwa auch Wolfgang Clement, der langjährige Ministerpräsident von Nordrhein-Westfalen sehr kümmerte. In NRW wollen sie immer noch die Defizite wegbasteln, die sie durch die Umbruchlandschaft der leeren Kohlegruben haben. Insofern kann man schnell glauben, fast alle deutschen Komiker kämen aus NRW, der rheinische Frohsinn wäre dominierend. Wenn man sich in der Berliner LiveKultur umschaut, sieht man, dass es anders ist. Es sind ja auch einige Größen von hier in der kommerziellen Kultur angekommen, wie Kurt Krömer oder Cindy aus Marzahn, die sich trotz aller Kommerzialität vom Rest unterscheiden. Berlin ist die Kulturlandschaft, in der sich jeder selbst leben kann, geduldet wird und keine Anpassung verlangt wird. Das entzieht sich auch als Werbefolien ein bisschen, deshalb ist das auch nicht so leicht integrierbar. Und dies eben nicht nur, weil hier nicht so viele Produktionsfirmen sitzen. Berlin ist einfach immer noch die Stadt der Verrückten und Daneben-Lebenden. Das macht auch die ungeheure Attraktivität aus. Alle Leute wollen nach Berlin. Deutschland steht ja eigentlich für das, was aus Schwaben kommt, dieses Pietistische, dieses Calvinistische, dieses Pünktliche, vielleicht auch Preußische, früher noch dieses Genaue: Made in Germany ${ }^{10}$. Alle Jugendlichen kommen hierher, nach Berlin, auch die jungen Schwaben werden in Berlin sozialisiert. Die erleben hier seit Jahrzehnten ihre „alternative Armeezeit“. Das ist auch die kulturelle Aufgabe von Berlin: zu zeigen, dass es ein schräges Bewusstsein gibt. Hier ist die Hauptstadt des integrierten höheren Blödsinns. Und das gilt nicht nur für die Regierung. Berlin war einmal die größte Industriestadt Europas und ist heute die Stadt mit dem größten Defizit, den wenigsten „ordentlichen“ Arbeitsplätzen. Aber nicht nur durch die interessante Mietstruktur zieht die Stadt viele, viele außergewöhnliche Leute an, was sicher sehr fruchtbar für diese Gesellschaft ist. Das wird sich hoffentlich in einigen Jahrzehnten zeigen. Gemäß dem Satz von George Bernhard Shaw: „Was wir brauchen, sind ein paar verrückte Leute. Wir sehen doch, wohin uns die Normalen gebracht haben.“

AH: Arnulf Rating, ich bedanke mich für das Gespräch. 